\title{
Management of the vertebral artery during thoracic endovascular aortic repair with coverage of the left subclavian artery
}

\author{
Jian Zhu* Er-Ping Xi*, Shui-Bo Zhu, Gui-Lin Yin, Rong-Ping Wang, Yu Zhang \\ Department of Thoracic Cardiovascular Surgery, Wuhan General Hospital of the People's Liberation Army, Wuhan 430070, China \\ Contributions: (I) Conception and design: SB Zhu; (II) Administrative support: SB Zhu, GL Yin, RP Wang; (III) Provision of study materials or \\ patients: J Zhu, EP Xi, Y Zhang; (IV) Collection and assembly of data: J Zhu, EP Xi; (V) Data analysis and interpretation: J Zhu, EP Xi; (VI) \\ Manuscript writing: All authors; (VII) Final approval of manuscript: All authors. \\ *These authors contributed equally to this work. \\ Correspondence to: Shui-Bo Zhu, MD, PhD. Department of Thoracic Cardiovascular Surgery, Wuhan General Hospital of the People's Liberation \\ Army, \#627, Wuluo Rd., Wuchang District, Wuhan 430070, China. Email: zhudandan2008@163.com.
}

Background: The application of thoracic endovascular aortic repair (TEVAR), a minimally invasive operation, in the aortic arch has been a challenge of cardiovascular surgery in recent years. This study aimed to investigate management of the vertebral artery with coverage of the left subclavian artery (LSA) during TEVAR.

Methods: From January 2007 to September 2014 in the Department of Cardiothoracic Surgery at Wuhan General Hospital of Guangzhou Military Region, 160 patients underwent LSA closure or partial coverage during TEVAR of an aortic lesion near the LSA. The vertebral artery treatment, the reason for the surgical approach selection, and the prognosis were analyzed.

Results: In 94 patients with partial LSA coverage during TEVAR, no treatment was provided for the vertebral arteries, revealing blood flow of the left vertebral artery forward into the skull after surgery. For 66 patients with full LSA coverage (closure) during TEVAR, right carotid artery-left common carotid artery bypass surgery was performed before TEVAR in ten patients, without any treatment for the vertebral artery, showing reverse blood flow of the left vertebral artery after surgery. Left common carotid artery-LSA bypass surgery was performed before TEVAR in four patients; right common carotid artery-left common carotid artery-LSA bypass surgery was performed before TEVAR in three cases, and 6 out of these 7 patients underwent proximal LSA ligation, showing no obvious blood flow in the left vertebral artery. The closure of the LSA aortic arch opening using an occluder was performed in one patient, preserving the forward blood flow in the left vertebral artery. Among the 160 patients in this study, postoperative recurrent laryngeal nerve injury occurred in one patient after right common carotid artery-left common carotid artery-LSA bypass surgery, and the remaining 159 patients had no significant severe complications or death within 1 postoperative month.

Conclusions: Appropriate management of the aortic arch branch vessels may expand the application of TEVAR to the aortic arch and reduce complications, especially for high-risk patients who have a difficult time tolerating thoracotomy.

Keywords: Aortic dissection; endovascular repair; aortic arch; left subclavian artery (LSA); vertebral artery

Submitted Dec 11, 2016. Accepted for publication Mar 13, 2017.

doi: $10.21037 /$ jtd.2017.04.27

View this article at: http://dx.doi.org/10.21037/jtd.2017.04.27 


\section{Introduction}

Thoracic aortic dissection or aneurysm is devastating and life-threatening. If active and effective treatment is not available, morbidity and mortality rates can be high $(1,2)$. In recent years, thoracic endovascular aortic repair (TEVAR), as a minimally invasive means to treat thoracic aortic dissection or aneurysm, has been rapidly developed $(3,4)$. However, when the rupture involves the aortic branch vessels, the application of TEVAR may cause closure of the branch vessels and severe complications, such as left upper limb ischemia, posterior circulation ischemia, and even cerebral infarction $(3,5)$. How to accurately perform the preoperative anatomical assessment of the aortic branch vessel and to develop a personalized surgical plan, with sophisticated branch vessel management, is the key to break through the "bottle-neck" of this technology. In this study, based on single-center clinical experience, treatment of the vertebral artery with closure of the left subclavian artery (LSA) in aortic endovascular repair was retrospectively evaluated.

\section{Methods}

\section{Clinical data}

From January 2007 to September 2014, 160 patients underwent LSA closure or partial coverage during TEVAR of an aortic lesion near the LSA in the Department of Cardiothoracic Surgery at Wuhan General Hospital of the Guangzhou Military Region. Of the included patients, 93 were male, and the mean age was $55.3 \pm 15.2$ years. There were 60 cases of type A aortic dissection (one case of left vertebral artery originating from the aortic arch with type A aortic dissection, five cases of proximal inverse tear after TEVAR), 81 cases of type B aortic dissection (one case of left vertebral artery originating from the aortic arch with type B aortic dissection, one case of proximal inverse tear after TEVAR; 15 cases of complicated and 66 cases of uncomplicated type B aortic dissection), 19 cases of pseudoaneurysm.

The vessel stent, delivery system, and guidewire used in this study were from LifeTech (Shenzhen) Scientific Co., Ltd. (Shenzhen, China). A polytetrafluoroethylene artificial vessel with a Gore-Tex inner supporting ring with an 8-mm diameter (W.L. GORE \& ASSOCIATES, INC. Arizona, the United States) was used in the aortic-arch-branch-vessel bypass surgery.

\section{Preoperative evaluation}

All patients receiving TEVAR underwent a preoperative computed tomographic angiography (CTA) examination. The location of the aortic lesion and its positional relation to the opening portion of the LSA were determined by intraoperative digital subtraction angiography (DSA) during TEVAR. The conditions of the cerebral circulation, carotid artery, basilar artery, and circle of Willis were assessed. The following cases required surgical treatment for the vertebral artery: (I) if the circle of Willis was intact, with good blood supply in the right vertebral artery, and the left vertebral artery showed no obvious weaknesses or originated from the aortic arch, then the LSA and left vertebral artery could be directly closed as necessary; (II) if the circle of Willis was intact and the right vertebral artery showed an obvious advantage, then the balloon occlusion test was required for the left vertebral artery; if the result was negative, the left vertebral artery could be closed; if the result was positive, bypass surgery was necessary to ensure blood flow of the left vertebral artery forward into the skull; and (III) if the circle of Willis was intact and the left vertebral artery showed an obvious advantage, then bypass surgery was necessary to ensure blood flow of the left vertebral artery forward into the skull (no such cases in this study). The following cases did not require surgical treatment for the vertebral artery: (I) no special contraindication was shown for partial coverage of the LSA; (II) the circle of Willis was intact, with good blood supply in the right vertebral artery, and the left vertebral artery showed no significant weaknesses affecting LSA closure; and (III) the circle of Willis was intact, with a good blood supply in the right vertebral artery, and the left vertebral artery showed no obvious weaknesses affecting aortic-arch-branch-vessel bypass surgery (hybrid procedure).

\section{Treatment procedures}

For the cases with stable conditions and no progression of a false lumen (based on the CTA results), surgery was performed after 2 weeks of conservative treatment since onset $(n=139)$. During the conservative treatment, if the patient experienced emergency situations such as distal limb ischemia, unalleviated severe tearing chest and back pain, abnormal consciousness, high blood pressure that was difficult to control, and poor splanchnic vascular perfusion (no urine, intestinal paralysis, etc.), emergency surgery was 


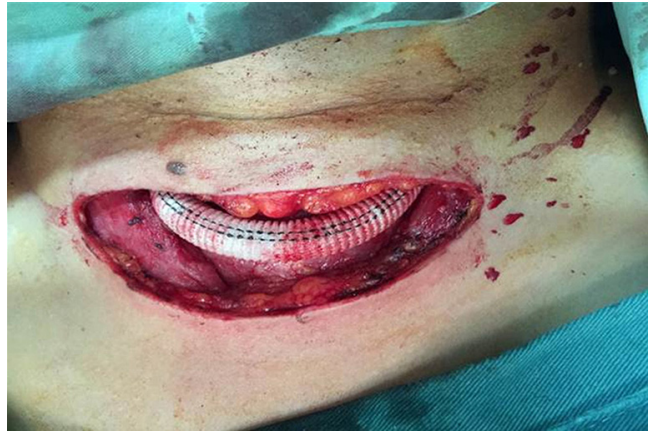

Figure 1 Right common carotid artery-left common carotid artery bypass surgery before TEVAR. TEVAR, thoracic endovascular aortic repair.

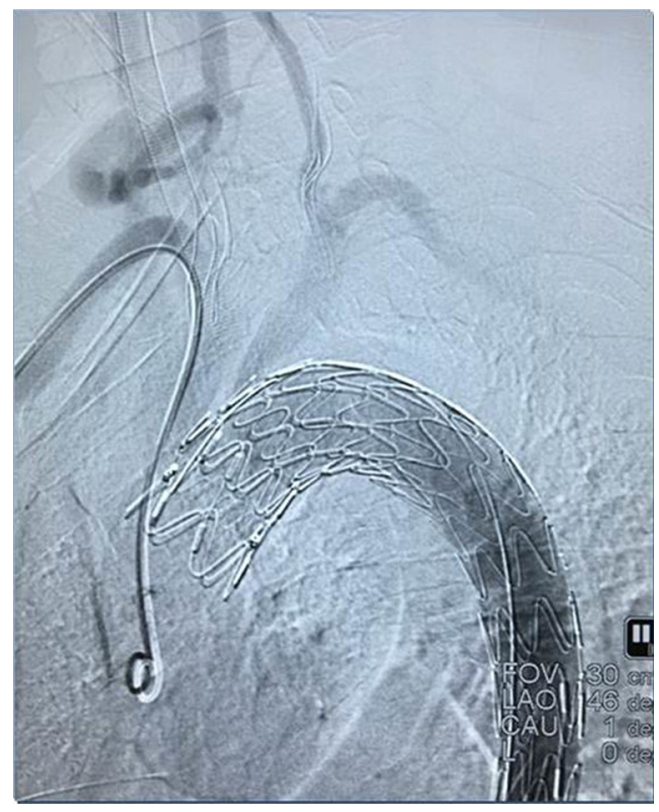

Figure 2 Right common carotid artery-left common carotid artery bypass surgery was performed before TEVAR, without any treatment for the vertebral artery, showing reverse blood flow of the left vertebral artery after the surgery.

performed ( $\mathrm{n}=21)$.

General anesthesia or local anesthesia was administered for the surgery, with radial artery puncture on the left and right sides for monitoring blood pressure. The aorticarch-branch-vessel bypass surgery was implemented in the operating room, and the timing for TEVAR in the catheterization laboratory was determined based on the patient's conditions. The post-releasing tapered coated graft stent with a bare stent produced by LifeTech (Shenzhen)
Scientific Co., Ltd., was used during TEVAR. The procedures for the aortic-arch-branch-vessel bypass were (I) occlusion of the right common carotid artery, followed by anastomosis of the right common carotid artery-artificial vessel and opening of the right common carotid artery; (II) occlusion of the left common carotid artery, followed by anastomosis of the artificial vessel-left common carotid artery and opening of the left common carotid artery; and (III) occlusion of the LSA, followed by anastomosis of the artificial vessel-LSA and opening of the LSA. The anastomosis of the artificial vessel and the vascular branch was conducted using a 6-0 prolene suture. TEVAR was performed using conventional methods.

After surgery, the patient was sent to the ICU of our department. The patient was given treatments for blood pressure and heart rate control and pain relief. Seven days after surgery, the aorta was examined by CTA. The patient was discharged after confirming good repair of the aortic rupture and a normal position and morphology of the stent. At 3, 6, and 12 months after surgery and once a year thereafter, the aorta was examined by CTA.

\section{Results}

Among the 160 patients in this study, partial LSA coverage by TEVAR was implemented in 94 patients because the aortic rupture did not involve the LSA, without any treatment for the vertebral arteries. No patients complained of vertigo or other symptoms of posterior circulation ischemia, exhibiting blood flow of the left vertebral artery forward into the skull after surgery. LSA closure during TEVAR was required for 66 patients, of whom 64 showed an intact circle of Willis, good blood supply in the right vertebral artery, and no obvious weakness in the left vertebral artery. Direct closure of the LSA was necessary due to TEVAR (right common carotid artery-left common carotid artery bypass surgery before TEVAR was performed in ten cases, as shown Figure 1), with no treatment for the vertebral artery, showing reverse blood flow in the left vertebral artery after surgery (Figure 2). Due to the obvious weakness of the left vertebral artery in two cases, a balloon occlusion test was performed for the left vertebral artery (Figure 3). The results were negative, suggesting that direct LSA closure was necessary due to TEVAR. Mild clinical symptoms occurred in some of these 66 patients, but no further surgical intervention was required. Before TEVAR, left common carotid artery-LSA bypass surgery was performed in four patients (Figure 4), and right common 


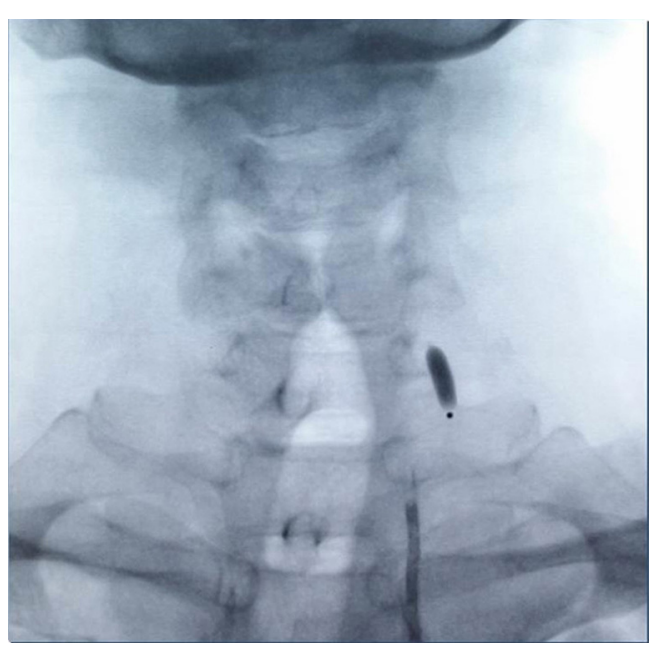

Figure 3 Balloon occlusion test for the vertebral artery.

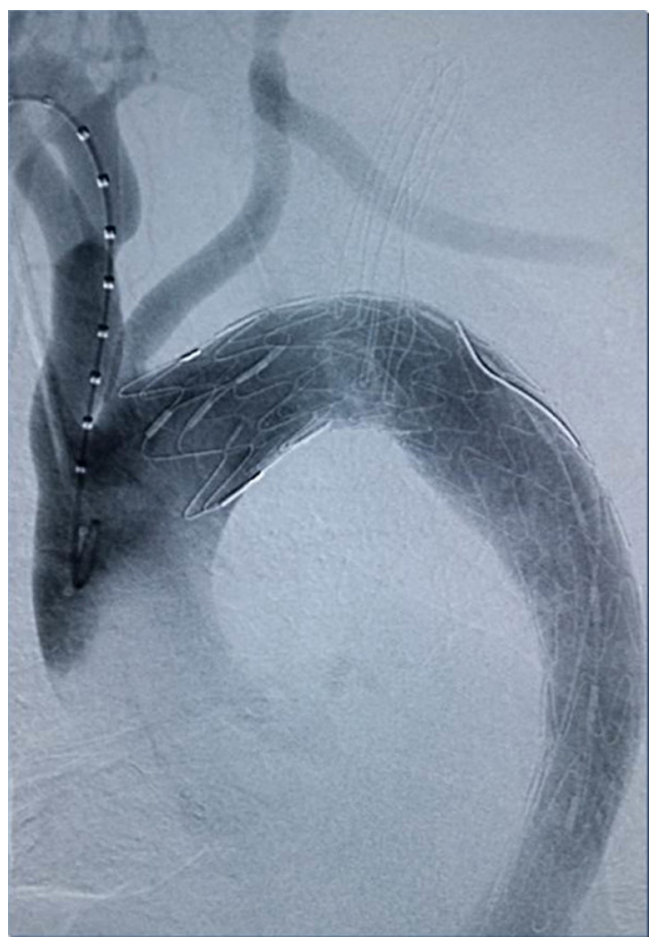

Figure 4 Preoperative left common carotid artery-LSA bypass surgery was performed, showing forward blood flow in the LSA after TEVAR. LSA, left subclavian artery.

carotid artery-left common carotid artery-LSA bypass surgery was performed in three patients (Figure 5); 6 of these 7 patients underwent proximal LSA ligation, showing no obvious blood flow in the left vertebral artery. A balloon occlusion test for the left vertebral artery was conducted

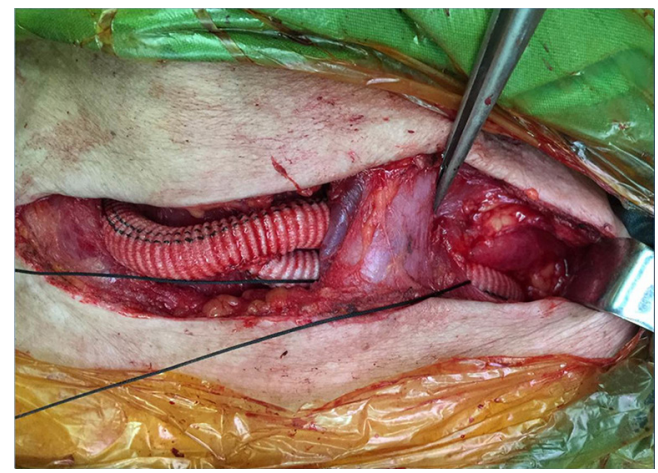

Figure 5 Right common carotid artery-left common carotid artery-LSA bypass surgery before TEVAR.

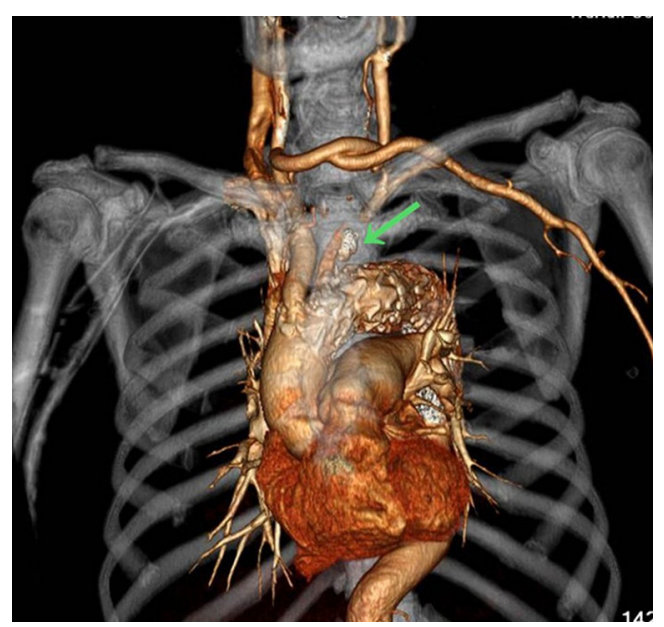

Figure 6 Right common carotid artery-left common carotid artery-LSA bypass surgery was performed before TEVAR. The result of the balloon occlusion test of the left vertebral artery was positive. The closure of the LSA aortic arch opening was performed during TEVAR using a PDA occluder (arrowed), showing LSA bypass blood flow forward into the skull through the left vertebral artery after surgery. PDA, patent ductus arteriosus.

in one patient, and the result was positive; a patent ductus arteriosus (PDA) occluder [LifeTech (Shenzhen) Scientific Co., Ltd.] was used to close the opening of the LSA aortic arch, resulting in LSA bypass blood flow forward through the left vertebral artery into the skull (Figure 6). Among the 160 patients in this study, postoperative aphonia and choking occurred in one patient after right common carotid artery-left common carotid artery-LSA bypass surgery, and the remaining 159 patients had no significant severe complications (Table 1). No patient died within 1 month after surgery. Postoperative CT scanning was performed 
Table 1 General clinical information of the patients with LSA treatment

\begin{tabular}{|c|c|c|}
\hline Treatment & Operations & Complications \\
\hline \multirow[t]{9}{*}{ Closure of the LSA } & \multirow{2}{*}{$\begin{array}{l}\text { Closure of LSA only with no other } \\
\text { operation }(n=46) \text { : aortic dissection } \\
(n=42), \text { pseudoaneurysm }(n=4)\end{array}$} & Paleness of the left upper limb, with low skin temperature $(n=8)$ \\
\hline & & Pain and numbness of the left upper limb after exercising $(n=8)$ \\
\hline & \multirow{2}{*}{$\begin{array}{l}\text { Negative in the preoperative left } \\
\text { vertebral artery balloon occlusion test } \\
(n=1) \text { : aortic dissection }(n=1)\end{array}$} & Paleness of the left upper limb, with low skin temperature $(n=1)$ \\
\hline & & Paroxysmal vertigo $(n=1)$ \\
\hline & $\begin{array}{l}\text { Closure of the left vertebral artery } \\
(n=2) \text { : the left vertebral artery originated } \\
\text { from the aortic arch }(n=2)\end{array}$ & Paleness of the left upper limb, with low skin temperature $(n=1)$ \\
\hline & \multirow{4}{*}{$\begin{array}{l}\text { Right carotid artery-left common } \\
\text { carotid artery bypass surgery }(n=10) \text { : } \\
\text { aortic dissection }(n=7) \text {; Proximal } \\
\text { inverse tear after TEVAR }(n=3)\end{array}$} & Paleness of the left upper limb, with low skin temperature $(n=2)$ \\
\hline & & Pain and numbness of the left upper limb after exercising $(n=1)$ \\
\hline & & Intermittent muscle weakness in the left upper limb $(n=3)$ \\
\hline & & Paroxysmal vertigo $(n=2)$ \\
\hline \multirow[t]{2}{*}{ LSA bypass reconstruction } & $\begin{array}{l}\text { Left common carotid artery-LSA } \\
\text { bypass surgery }(n=4) \text { : aortic dissection } \\
(n=2) \text {; proximal inverse tear after } \\
\text { TEVAR }(n=2)\end{array}$ & $\begin{array}{l}\text { No clinical complications of the left upper limb and posterior } \\
\text { circulation }\end{array}$ \\
\hline & $\begin{array}{l}\text { Right common carotid artery-left } \\
\text { common carotid artery-LSA bypass } \\
\text { surgery* }(n=3) \text { : aortic dissection }(n=2) \text {; } \\
\text { proximal inverse tear after TEVAR }(n=1)\end{array}$ & Aphonia, choking $(n=1)$ \\
\hline
\end{tabular}

\#, the patients' left vertebral arteries originate from the aortic arch and near the LSA; *, one patient in this group showed a positive result in the preoperative left vertebral artery balloon occlusion test, this patient did not have related postoperative complications in the left upper limb and posterior circulation. LSA, left subclavian artery; TEVAR, thoracic endovascular aortic repair.

7 days, 3 months, 1 year, and once a year thereafter. Within 1 year, all patients had survived in a healthy condition, with unobstructed bypass artificial vessels and no endoleaks in the TEVAR stent.

\section{Discussion}

Thoracic aortic dissection or aneurysm is a devastating and life-threatening disease (6). If active and effective treatment is not available, morbidity and mortality rates can be high. Although thoracotomy for aortic arch replacement has been well implemented in clinical application and promotion and has achieved good clinical efficacy, the procedure shows a high incidence of perioperative paraplegia and stroke and even a high mortality because of its requirement for deep hypothermic circulatory arrest and selective cerebral perfusion $(7,8)$. Therefore, the procedure is not suitable for elderly patients with complications in the respiratory tract and cardiovascular system. The development of TEVAR, a less invasive technique, has resulted in reduced operative time and length of hospital stay, improved perioperative morbidity, and mortality rates as low as $2.1 \%(9)$. As the utilization of TEVAR has increased, it has been applied to a wider range of pathologies, including dissections, and traumatic aortic aneurysm $(3,10)$.

It has also been applied to break through the restricted area in the aortic arch for open surgery $(11,12)$. However, due to its special anatomical location, the aortic arch artery involves three major blood vessels on the arch: the innominate artery, the left common carotid artery and 
the left subclavian artery, making TEVAR of aortic arch diseases one of the most challenging research projects in the current cardiovascular surgery field. The main reason is that successful TEVAR requires a sufficient length of normal vessel wall at the proximal and distal ends of the aortic rupture to ensure its adequate bonding with the stent. The vessel wall is called the landing zone (LZ), and sufficient length of the proximal $\mathrm{LZ}$ is a prerequisite for TEVAR $(4,13)$. The proximal LZ beyond the LSA is estimated to be of inadequate length in $26-40 \%$ of patients with disease of the descending thoracic aorta $(14,15)$. In these cases, covering the LSA is a common technique during TEVAR treatment of aortic arch rupture disease (16).

In 2010, the Society for Vascular Surgery (SVS) published updated recommendations regarding coverage of the LSA during TEVAR. These recommendations noted that in non-urgent cases, the LSA should be revascularized before TEVAR and that in urgent cases, the decision to revascularize can be made after TEVAR is performed (14). LSA coverage has often caused complications, such as left upper limb ischemia, posterior circulation ischemia, stroke, or spinal cord ischemia. Therefore, the reconstruction of the aortic arch branch vessels must achieve the desired circular path. Based on current technology, how to manage the LSA and vertebral artery is the key to the success of this technology. With good management of the LSA and vertebral artery, most centers of intervention can apply TEVAR to the rupture, the involved right common carotid artery, and even closer to the brachiocephalic trunk $(17,18)$.

The LSA after the treatment revealed three situations: partial coverage, full coverage, and full coverage with bypass reconstruction. In our experience, the symptoms of left upper limb ischemia and posterior circulation ischemia did not occur in the patients with partial LSA coverage. The risks of full LSA coverage include two aspects: (I) the left arm controlled by the left axillary artery as the main branch of the LSA and (II) the posterior circulation controlled by the left vertebral artery as an important branch of the LSA, in which reconstruction of the left vertebral artery bypass is more complicated and the consequences of the complications caused by closure of the left vertebral artery are more serious.

In the patients with LSA closure, the left axillary arteries mostly exhibited weak forward blood flow from the left vertebral artery through the arterial bypass of the circle of Willis. However, for patients receiving aortic coronary artery bypass graft surgery using the left internal mammary artery (LIMA), with severe stenosis of the carotid artery or right vertebral artery, functional defects in the circle of Willis, and subclavian artery anatomic variation, neurological complications and blood steal symptoms in the left upper limb would easily occur; consequently, left common carotid artery-LSA bypass surgery should be conducted. Compared with the extensive trauma and the related high expense of open surgery, left common carotid artery-LSA bypass surgery is a minimally invasive surgical procedure with relatively mature technology and a low cost. Thus, the complications caused by LSA closure may be easily avoided.

However, the left vertebral artery originating from the LSA is a major component of the vertebral basilar artery and is responsible for $2 / 5$ of the blood supply of the posterior cerebral artery (19). Compared with the brain, no other organs are more vulnerable to irreversible insufficient blood supply. Therefore, good management of the vertebral artery is the key to the success of this type of surgery. If the circle of Willis is intact, the blood supply of the right vertebral artery is good, and the left vertebral artery has no obvious weakness, direct closure of the left vertebral artery when necessary poses no obvious contraindications to surgery $(20,21)$. If the right vertebral artery shows an obvious advantage, balloon test occlusion (BTO) of the left vertebral artery is necessary when closure of the left vertebral artery is needed. If the result is negative, suggesting that closure of the left vertebral artery has no significant effect, then closure of the left vertebral artery can be performed during TEVAR $(22,23)$. A positive result indicates that the left vertebral artery cannot be closed. Closure of the opening at the LSA root with preserved blood flow of the left vertebral artery is difficult to achieve by carotid artery bypass surgery, and thoracotomy is often required. After analyzing the anatomical characteristics of the aortic arch branch vessel, this problem can be solved by implementing the left common carotid artery-LSA bypass surgery, with PDA occluder placement at the proximal end of the left common carotid artery, thus avoiding thoracotomy and achieving closure of the LSA root opening while ensuring blood flow of the left vertebral artery forward into the skull. This method can also be applied to cases with a left vertebral artery advantage and coverage of the LSA-that is, minimally invasive reconstruction of the left vertebral artery with blood flowing forward into the skull at the same time of LSA closure-is considered not applicable $(24,25)$.

Among the included cases in this study, postoperative aphonia and choking occurred in one patient after the implementation of right common carotid artery-left 
common carotid artery-LSA bypass surgery. We believe this event is related to irreversible damage of the recurrent laryngeal nerve while dissociating the left common carotid artery during the bypass surgery instead of a posterior circulatory disorder caused by the vertebral artery closure.

In conclusion, the left common carotid artery, LSA, and left vertebral artery on the aortic arch can be reconstructed using minimally invasive procedures without thoracotomy. The appropriate treatment for the aortic arch branch vessels can expand the application range of TEVAR in the aortic arch, with reduced complications.

\section{Acknowledgements}

Funding: This work was supported by grants from the Application and Basic Research Program of the Wuhan Science and Technology Bureau (No. 2015060101010053), 2010 military clinical high-tech funded plan projects (No. 2010 gxjs036) and the Wuhan General Hospital of Guangzhou Command funded plan (No. YZ201401).

\section{Footnote}

Conflicts of Interest: The authors have no conflicts of interest to declare.

Ethical Statement: The study was approved by institutional ethics committee board of the Wuhan General Hospital of the People's Liberation Army (No. 2010gxjs036) and was consistent with the principles outlined in the Declaration of Helsinki. Written informed consent was obtained from the patient for publication of this manuscript and any accompanying images.

\section{References}

1. Dake MD, Miller DC, Semba CP, et al. Transluminal placement of endovascular stent-grafts for the treatment of descending thoracic aortic aneurysms. N Engl J Med 1994;331:1729-34.

2. Xi EP, Zhu J, Zhu SB, et al. Secondary aortoesophageal fistula after thoracic aortic aneurysm endovascular repair: literature review and new insights regarding the hypothesized mechanisms. Int J Clin Exp Med 2014;7:3244-52.

3. Zamor KC, Eskandari MK, Rodriguez HE, et al. Outcomes of Thoracic Endovascular Aortic Repair and Subclavian Revascularization Techniques. J Am Coll Surg
2015;221:93-100.

4. Lee TC, Andersen ND, Williams JB, et al. Results with a selective revascularization strategy for left subclavian artery coverage during thoracic endovascular aortic repair. Ann Thorac Surg 2011;92:97-102; discussion 102-3.

5. Ren C, Guo X, Sun L, et al. One-stage hybrid procedure without sternotomy for treating thoracic aortic pathologies that involve distal aortic arch: a single-center preliminary study. J Thorac Dis 2015;7:861-7.

6. Ramanath VS, Oh JK, Sundt TM 3rd, et al. Acute aortic syndromes and thoracic aortic aneurysm. Mayo Clin Proc 2009;84:465-81.

7. Sun L, Qi R, Zhu J, et al. Total arch replacement combined with stented elephant trunk implantation: a new "standard" therapy for type a dissection involving repair of the aortic arch? Circulation 2011;123:971-8.

8. Higashi R, Matsumura Y, Yamaki F. A single stage hybrid repair of a complicated acute type B dissection with aortic arch involvement. Ann Vasc Dis 2014;7:141-4.

9. Hoel AW. Aneurysmal disease: thoracic aorta. Surg Clin North Am 2013;93:893-910, ix.

10. Peterson BG, Eskandari MK, Gleason TG, et al. Utility of left subclavian artery revascularization in association with endoluminal repair of acute and chronic thoracic aortic pathology. J Vasc Surg 2006;43:433-9.

11. Patterson B, Holt $P$, Nienaber C, et al. Aortic pathology determines midterm outcome after endovascular repair of the thoracic aorta: report from the Medtronic Thoracic Endovascular Registry (MOTHER) database. Circulation 2013;127:24-32.

12. Howard DP, Banerjee A, Fairhead JF, et al. Populationbased study of incidence and outcome of acute aortic dissection and premorbid risk factor control: 10-year results from the Oxford Vascular Study. Circulation 2013;127:2031-7.

13. Klocker J, Koell A, Erlmeier M, et al. Ischemia and functional status of the left arm and quality of life after left subclavian artery coverage during stent grafting of thoracic aortic diseases. J Vasc Surg 2014;60:64-9.

14. Matsumura JS, Lee WA, Mitchell RS, et al. The Society for Vascular Surgery Practice Guidelines: management of the left subclavian artery with thoracic endovascular aortic repair. J Vasc Surg 2009;50:1155-8.

15. Matsumura JS, Rizvi AZ, Society for Vascular Surgery. Left subclavian artery revascularization: Society for Vascular Surgery Practice Guidelines. J Vasc Surg 2010;52:65S-70S.

16. Zhang L, Lu Q, Zhou J, et al. Alternative management of the left subclavian artery in thoracic endovascular aortic 
repair for aortic dissection: a single-center experience. Eur J Med Res 2015;20:57.

17. Scali ST, Chang CK, Pape SG, et al. Subclavian revascularization in the age of thoracic endovascular aortic repair and comparison of outcomes in patients with occlusive disease. J Vasc Surg 2013;58:901-9.

18. Walsh SR, Tang TY, Sadat U, et al. Endovascular stenting versus open surgery for thoracic aortic disease: systematic review and meta-analysis of perioperative results. J Vasc Surg 2008;47:1094-8.

19. Woo EY, Bavaria JE, Pochettino A, et al. Techniques for preserving vertebral artery perfusion during thoracic aortic stent grafting requiring aortic arch landing. Vasc Endovascular Surg 2006;40:367-73.

20. Cinà CS, Safar HA, Laganà A, et al. Subclavian carotid transposition and bypass grafting: consecutive cohort study and systematic review. J Vasc Surg 2002;35:422-9.

21. Gutsche JT, Cheung AT, McGarvey ML, et al.

Cite this article as: Zhu J, Xi EP, Zhu SB, Yin GL, Wang RP, Zhang Y. Management of the vertebral artery during thoracic endovascular aortic repair with coverage of the left subclavian artery. J Thorac Dis 2017;9(5):1273-1280. doi: 10.21037/ jtd.2017.04.27
Risk factors for perioperative stroke after thoracic endovascular aortic repair. Ann Thorac Surg 2007;84:1195-200; discussion 1200.

22. Kai Y, Hamada J, Morioka M, et al. Dissecting aneurysms of the vertebral artery--angiographic patterns at the dissecting site on balloon test occlusion. Neuroradiology 2012;54:857-62.

23. Palchik E, Bakken AM, Wolford HY, et al. Subclavian artery revascularization: an outcome analysis based on mode of therapy and presenting symptoms. Ann Vasc Surg 2008;22:70-8.

24. Woo EY, Carpenter JP, Jackson BM, et al. Left subclavian artery coverage during thoracic endovascular aortic repair: a single-center experience. J Vasc Surg 2008;48:555-60.

25. Kotelis D, Geisbüsch P, Hinz U, et al. Short and midterm results after left subclavian artery coverage during endovascular repair of the thoracic aorta. J Vasc Surg 2009;50:1285-92. 